Relations industrielles

Industrial Relations

\title{
Directives aux patrons catholiques
}

\section{Pie XII}

Volume 4, numéro 9, mai 1949

URI : https://id.erudit.org/iderudit/1023963ar

DOI : https://doi.org/10.7202/1023963ar

Aller au sommaire du numéro

\section{Éditeur(s)}

Département des relations industrielles de l’Université Laval

\section{ISSN}

0034-379X (imprimé)

1703-8138 (numérique)

Découvrir la revue

\section{Citer cet article}

Pie XII (1949). Directives aux patrons catholiques. Relations industrielles /

Industrial Relations, 4(9), 81-83. https://doi.org/10.7202/1023963ar

Tous droits réservés @ Département des relations industrielles de l’Université Laval, 1949
Ce document est protégé par la loi sur le droit d'auteur. L’utilisation des services d'Érudit (y compris la reproduction) est assujettie à sa politique d'utilisation que vous pouvez consulter en ligne.

https://apropos.erudit.org/fr/usagers/politique-dutilisation/ 


\section{Bulletin des relations industrielles}

Publié par le

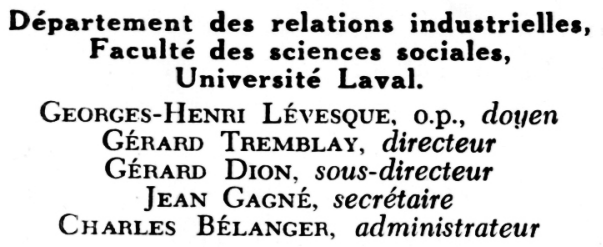

Le Bulletin paraît mensuellement de septembre à juin (dix numéros par année). Abonnement annuel: Canada: $\$ 2.00$; étran-

ger: \$2.50. Vingt-cinq cents le numéro. Publication répertoriée dans le "Canadian Index".

\section{Adressez toute correspondance au secrétaire de rédaction Gérard Dion \\ 2, rue de l'Université, Québec.}

Bulletin des relations industrielles

Volume 4 , numéro 9 Mai 1949

\section{Sommaire}

Directives aux patrons catholiques S.S. PIE XII

La rupture du contrat de travail Georges-Michel Giroux

Le congé des salariés pour voter à une élection

Statistiques des relations de travail

Thèses présentées pour l'obtention de la maitrise en relations industrielles.

L'Eglise et le capitalisme

Comte della Torre

\section{Collaborateurs}

Archambault, Jacques, B.A., M.Sc.S., secrétaire du Conseil central des syndicats nationaux catholiques de Joliette.

Grroux, Georges-Michel, N.P., LL.D., conseiller technique à la Commission du salaire minimum et professeur aux Facultés de droit et de sciences sociales de Laval.

\section{DIRECTIVES AUX PATRONS CATHOLIQUES}

\author{
Texte de l'allocution de S.S. Pie XII à l'occasion \\ de l'audience accordée aux associations patronales \\ chrétiennes (UNI. PAC), l'A.P.I. étant \\ représentée, le 7 mai 1949.
}

Avec une égale sollicitude, un égal intérêt, Nous voyons venir à Nous, tour à tour, les ouvriers et les représentants des organisations industrielles; les uns et les autres Nous exposent, avec une confiance qui Nous touche profondément leurs préoccupations respectives. Aussi, en vous souhaitant de tout coeur la bienvenue, Nous saisissons volontiers, très chers fils, l'occasion que vous nous offrez de vous exprimer Notre paternelle bienveillance et de louer votre zèle à faire pénétrer dans le monde de l'économie la doctrine sociale chrétienne.

Nous venons de faire allusion aux préoccupations de ceux qui participent à la production industrielle. Erroné et funeste en ses conséquences est le préjugé, malheureusement trop répandu, qui voit en elles une opposition irréductible d'intérêts divergents. L'opposition n'est qu'apparente. Dans le domaine économique il y a communauté d'activité et d'intérêt entre chefs d'entreprises et ouvriers. Méconnaître ce lien réciproque, travailler à le briser, ne peut être que le fait d'une prétention de despotisme aveugle et déraisonnable. Chefs d'entreprises et ouvriers ne sont pas antagonismes inconciliables. Ils sont coopérateurs dans une oeuvre commune. Ils mangent, pour ainsi dire, à la même table, puisqu'ils vivent en fin de compte du bénéfice net et global de l'économie nationale. Chacun touche son revenu, et sous ce rapport leurs relations mutuelles ne mettent aucunement les uns au service des autres.

Toucher son revenu est un apanage de la dignité personnelle de quiconque, sous une forme ou sous une autre, comme patron ou comme ouvrier, prête son concours productif au rendement de l'économie nationale. Dans le bilan de l'industrie privée, la somme des salaires peut figurer à titre de frais de l'employeur. Mais, dans l'économie nationale, il n'est qu'une sorte de frais qui consistent dans les biens naturels utilisés en vue de la production nationale et qu'il faut, par conséquent, sans cesse suppléer. 
Il s'ensuit que, des deux côtés, on a intérêt à voir les dépenses de la production nationale proportionnelles à son rendement. Mais dès lors que l'intérêt est commun, pourquoi ne pourrait-il pas se traduire dans une expression commune? Pourquoi ne serait-il pas légitime d'attribuer aux ouvriers une juste part de responsabilité dans la constitution et le développement de l'économie nationale. Aujourd'hui surtout que la pénurie des capitaux, la difficulté des échanges internationaux paralysent le libre jeu des dépenses de la production nationale. Les récents essais de socialisation n'ont fait que mettre en plus claire évidence cett $\epsilon$ pénible réalité. Elle est un fait: ni la mauvaise volonté des uns ne l'a créé, ni la bonne volonté des autres ne réussira à l'éliminer. Mais alors, pourquoi, quand il est encore temps, ne pas mettre les choses au point, dans la pleine conscience de la commune responsabilité en sorte d'assurer les uns contre d'injustes défiances, les autres contre des illusions qui ne tarderaient pas à devenir un péril social ?

Cette communauté d'intérêt et de responsabilité dans l'oeuvre de l'économie nationale, Notre inoubliable Prédécesseur Pie XI en avait suggéré la formule concrète et opportune lorsque, dans son Encyclique «Quadragesimo Anno », il recommandait «l'organisation professionnelle» dans les diverses branches de la production. Rien, en effet, ne lui semblait plus propre à triompher du libéralisme économique que l'établissement, pour l'économie sociale, d'un statut de droit public fondé précisément sur la communauté de responsabilité entre tous ceux qui prennent part à la production. Ce point de l'Encyclique fut l'objet d'une levée de boucliers; les uns y voyaient une concession aux courants politiques modernes, les autres un retour au Moyen-Age. Il eut été incomparablement plus sage de déposer les vieux préjugés inconsistants et de se mettre de bonne foi et de bon coeur à la réalisation de la chose elle-même et des multiples applications pratiques.

Mais, à présent, cette partie de l'Encyclique semble presque nous fournir malheureusement un exemple de ces occasions opportunes qu'on laisse échapper, faute de les saisir à temps. Après coup, on s'évertue à élaborer d'autres formes d'organisation juridique publique de l'économie sociale et, pour le moment, la faveur va de préférence à l'étatisation et à la nationalisation des entreprises. Il n'est pas douteux que l'Eglise aussi - dans certaines justes limites - admet l'étatisation et juge «que l'on peut légitimement réserver aux pouvoirs publics certaines catégories de biens, ceux-là qui présentent une telle puissance, qu'on ne saurait, sans mettre en péril le bien commun, les abandonner aux mains des particuliers» (Encyclique «Quadragesimo Anno »- Acta Ap. Sedis, vol. 23, 1931, pag. 214). Mais faire de cette étatisation comme la règle normale de l'organisation publique de l'économie serait renverser l'ordre des choses. La mission du droit public est en effet de servir le droit privó non de l'absorber. L'économie pas plus d'ailleurs qu'aucune autre branche de l'activité humaine - n'est de sa nature une institution d'Etat; elle est, à l'envers, le produit vivant de la libre initiative des individus et de leur groupes librement constitués.

On ne serait pas non plus dans le vrai en voulant affirmer que toute entreprise particulière est par sa nature une société, de manière que les rapports entre participants y soient déterminés par les règles de la justice distributive, en sorte que tous indistinctement - propriétaires ou non des moyens de production - auraient droit à leur part de la propriété ou tout au moins des bénéfices de l'entreprise. Une telle conception part de l'hypothèse que toute entreprise rentre par nature dans la sphère du droit public. Hypothèse inexacte: que l'entreprise soit constituée sous forme de fondation ou d'association de tous les ouvriers comme co-propriétaires, ou bien qu'elle soit propriété privée d'un individu qui signe avec tous ses ouvriers un contrat de travail, dans un cas comme dans l'autre elle relève de l'ordre juridique privé de la vie économique.

Tout ce que nous venons de dire s'applique à la nature juridique de l'entreprise comme telle; mais l'entreprise peut comporter encore toute une catégorie d'autres rapports personnels entre participants, dont il faut aussi tenir compte, et même rapports de commune responsabilité. Le propriétaire des moyens de production, quel qu'il soit - propriétaire particulier, association d'ouvriers ou fondation - doit, toujours dans les limites du droit public de l'économie, rester maître de ses décisions économiques. Il va de soi que son revenu est plus élevé que celui de ses collaborateurs. Mais il s'ensuit que la prospérité matérielle de tous les membres du peuple, qui est le but de l'économie sociale, lui impose, à lui plus qu'aux autres, l'obligation de contribuer par l'épargne à l'accroissement du capital national. Comme il ne faut pas, d'autre part, perdre de vue qu'il est souverainement avantageux à une saine économie sociale que cet accroissement du capital provienne de sources 
aussi nombreuses que possible, il est par conséquent fort désirable que les ouvriers puissent eux aussi, du fruit de leur épargne, participer à la constitution du capital national.

Nombre d'hommes, industriels comme vous, catholiques, et non catholiques aussi, ont, en maintes circonstances, expressément déclaré que la doctrine sociale de l'Eglise - et elle seule - est en mesure de fournir les éléments essentiels pour une solution de la question sociale. Assurément la mise en oeuvre et l'application de cette doctrine ne peuvent être l'ouvrage d'un jour. Sa réalisation exige de tous les participants une sagesse clairvoyante et prévoyante, une forte dose de bon sens et de bon vouloir. Elle réclame d'eux surtout une réaction radicale contre la tentation de chercher chacun son propre avantage aux dépens des autres participants - quelle que soit la nature et la forme de leur participation - et au détriment du bien commun. Elle requiert enfin un désintéressement tel que, seule, une authentique vertu chrétienne, soutenue par l'aide et la grâce de Dieu, peut l'inspirer.

C'est pour attirer cette aide et cette grâce sur votre Union, sur son développement interne et sur son rayonnement au dehors, particulièrement dans les pays qui, tout catholiques qu'ils sont, ont pourtant besoin de s'ouvrir plus largement à la pensée sociale de l'Eglise, que Nous vous donnons, dans toute l'effusion de Notre coeur, à vous tous et à votre Association, sous le puissant patronage de la Mère du divin amour, Notre Bénédiction Apostolique.

\section{LA RUPTURE DU CONTRAT DE TRAVAIL}

\section{Georges-Michel Giroux}

L'abrogation de la Loi des maitres et des serviteurs (S.R.Q., 1941, c. 328), décrétée par le statut 13 Geo. VI, c. 69 , a modifié les règles sur la résiliation du contrat de travail. De multiples causes peuvent éteindre les obligations résultant de ce contrat, citons: l'accord de volontés, le dècès du salarié (C.c. a. 1668). La volonté unilatérale d'une des parties peut aussi y mettre fin, il y a alors rupture du contrat de travail.

Pour traiter de la rupture du contrat, il faut analyser les règles sur sa durée et celles sur le préavis de rupture, il faut aussi examiner le caractère des dispositions sur le délai-congé et l'effet de la réglementation du travail sur ce sujet.

\section{I - La durée du contrat}

Toute personne peut, quand il lui plaît, mettre fin à un contrat de travail qui la lie à une autre, c'est l'évidence même; un patron ne peut être forcé à embaucher un salarié et ce dernier, à travailler pour un employeur; nemo ad actum cogi potest. Le Conseil canadien des relations ouvrières, sous le régime de la Loi sur les relations industrielles et sur les enquêtes visant les différends du travail (Canada, 11-12 Geo. VI, c. 54), peut bien ordonner à un employeur de reprendre à son emploi des salariés congédiés indûment (a. 40,2); mais le patron, s'il ne se conforme pas à l'ordonnance, n'en- court que des pénalités (a. 40, 3). Un salarié, même sur l'ordre du Conseil canadien des relations ouvrières, ne peut travailler pour un employeur contre le gré de ce dernier.

Cependant le législateur a déterminé le temps où le contrat de travail pouvait être légalement rompu par la volonté d'une seule partie et a assuré à la partie lésée une indemnité quand l'autre partie en a provoqué abusivement la rupture. La loi, nous sommes ici en droit privé et non en droit public, veut assurer le respect de ce qui a été convenu; elle garantit la subsistance de l'engagement pour le terme, déterminé ou déterminable, fixé par les parties. L'étude sur la rupture du contrat de travail entraîne l'examen des règles légales sur la durée.

Les parties peuvent arrêter la durée de ce contrat:

a) pour une période déterminée (C.c. a. 1667 );

b) pour la durée d'une entreprise déterminée (Id.);

c) pour un temps indéterminé (Id. a. 1668);

d) à l'essai.

Les salariés dans la construction d'immeubles, s'engagent ordinairement pour la durée d'une entreprise déterminée; les briqueteurs louent leurs services pour le temps nécessaire à l'érection des murs. 